\title{
Applications of some fixed point theorems for fractional differential equations with Mittag-Leffler kernel
}

\author{
Hojjat Afshari ${ }^{1 *}$ and Dumitru Baleanu ${ }^{2,3}$
}

\section{"Correspondence:}

hojat.afshari@yahoo.com

'Department of Mathematics,

Faculty of Basic Science, University

of Bonab, Bonab, Iran

Full list of author information is

available at the end of the article

\section{Springer}

\begin{abstract}
Using some fixed point theorems for contractive mappings, including $\alpha-\gamma$-Geraghty type contraction, $\alpha$-type $F$-contraction, and some other contractions in $\mathcal{F}$-metric space, this research intends to investigate the existence of solutions for some Atangana-Baleanu fractional differential equations in the Caputo sense.

Keywords: Atangana-Baleanu derivative in the Caputo sense; $\mathcal{F}$-metric space; $\alpha$-type F-contractive mapping; Atangana-Baleanu fractional operator in the Caputo sense
\end{abstract}

\section{Introduction}

It is well known that several physical phenomena are described by nonlinear differential equations (both ODEs and PDEs). Therefore, the study of the many analytical and numerical methods used for solving the nonlinear differential equations is a very important topic for the analysis of engineering practical problems [1-19].

In 2016, the interesting and new derivatives without singular kernel were introduced by Atangana and Baleanu, which generalized the Caputo-Fabrizio definition [8]. AtanganaBaleanu derivative contains Mittag-Leffler function as a nonlocal and nonsingular kernel. Many authors showed their interest in this definition as it holds the profits of RiemannLiouville and Caputo derivatives [20-30]. Last year, Atangana et al. provided the numerical approximation to the fractional advection-diffusion equation whose fractional derivatives are Atangana-Baleanu derivative of Riemann-Liouville type [14].

In the last decades, two topics have been densely studied: "fixed point theory" and "fractional differential/integral equations". Recently, several significant results have been recorded [7, 31, 32].

In 2012, Samet et al. [33] studied the concept of $\alpha$-admissible mappings that was expanded by Karapınar and Samet in [34]. Also, Wardowski [35] proposed a new inequality to guarantee the existence and uniqueness of a given mapping in the framework of standard metric space. This inequality has been known as $F$-contraction.

(c) The Author(s) 2020. This article is licensed under a Creative Commons Attribution 4.0 International License, which permits use sharing, adaptation, distribution and reproduction in any medium or format, as long as you give appropriate credit to the original author(s) and the source, provide a link to the Creative Commons licence, and indicate if changes were made. The images or other third party material in this article are included in the article's Creative Commons licence, unless indicated otherwise in a credit line to the material. If material is not included in the article's Creative Commons licence and your intended use is not permitted by statutory regulation or exceeds the permitted use, you will need to obtain permission directly from the copyright holder. To view a copy of this licence, visit http://creativecommons.org/licenses/by/4.0/. 
In 2016, Gopal et al. considered new concepts of $\alpha$-type $F$-contractive mappings (see [12]). Very recently, Jleli and Samet [36] mentioned the concept of $\mathcal{F}$-metric space and obtained the generalization of Banach contraction principle.

In [1-4], the authors studied generalized Geraghty contractive mappings and their applications in $b$-metric spaces.

In this paper by applying some fixed point theorems for contractive mappings, like $\alpha$ $\gamma$-Geraghty type, $\alpha$-type $F$-contraction, and some other contractions in $\mathcal{F}$-complete $\mathcal{F}$ metric space, we study the existence of solutions for some Atangana-Baleanu fractional differential equations in the Caputo sense. Throughout the article $J$ denotes $[0,1]$.

Suppose that $(M, d)$ is a complete $b$-metric space (with constant $s_{1}$ ), also let $\Omega$ be a set of all increasing and continuous functions $\gamma:[0, \infty) \rightarrow[0, \infty)$ satisfying: $\gamma(c x) \leq c \gamma(x) \leq c x$ for all $c>1$, and $\Lambda$ is the family of all nondecreasing functions $\lambda:[0, \infty) \rightarrow\left[0, \frac{1}{s_{1}^{2}}\right), s_{1} \geq 1$.

Definition 1.1 ([2]) The mapping $g: M \rightarrow M$ is a generalized $\alpha-\gamma$-Geraghty contraction mapping whenever there exists $\alpha: M \times M \rightarrow[0, \infty)$ with

$$
\alpha(w, z) \gamma\left(s_{1}^{3} d(g w, g z)\right) \leq \lambda(\gamma(d(w, z))) \gamma(d(w, z))
$$

for $w, z \in M, \lambda \in \Lambda$, and $\gamma \in \Omega$

Definition $1.2([33])$ Let $\varphi: M \rightarrow M$, where $M$ is nonempty, and $\alpha: M \times M \rightarrow[0, \infty)$ be given, $g$ is $\alpha$-admissible if

$$
\alpha(w, z) \geq 1 \quad \Longrightarrow \quad \alpha(\varphi w, \varphi z) \geq 1, \quad \forall w, z \in M
$$

Theorem $1.3([2])$ Let $(M, d)$ be a complete $b$-metric space and $\varphi: M \rightarrow M$ be a generalized $\alpha-\gamma$-Geraghty contraction such that

(i) $\varphi$ is $\alpha$-admissible;

(ii) $\exists w_{0} \in M$ with $\alpha\left(w_{0}, \varphi w_{0}\right) \geq 1$;

(iii) $\left\{w_{n}\right\} \subseteq M, w_{n} \rightarrow u$ in $M$ and $\alpha\left(w_{n}, w_{n+1}\right) \geq 1$, then $\alpha\left(w_{n}, w\right) \geq 1$.

Then $\varphi$ has a fixed point.

Definition 1.4 ([8]) Let $\delta \in H^{1}(a, b), a<b$, and $0 \leq \kappa \leq 1$. The Atangana-Baleanu fractional derivative in the Caputo sense of $\delta$ of order $\kappa$ is defined by

$$
\left({ }_{a}^{A B C} D^{\kappa} \delta\right)(s)=\frac{B(\kappa)}{1-\kappa} \int_{a}^{s} \delta^{\prime}(\nu) E_{\kappa}\left[-\kappa \frac{(s-\nu)^{\kappa}}{1-\kappa}\right] d \nu,
$$

where $E_{\kappa}$ is the Mittag-Leffler function defined by $E_{\kappa}(z)=\Sigma_{n=0}^{\infty} \frac{z^{n}}{\Gamma(n \kappa+1)}$ and $B(\kappa)$ is a normalizing positive function satisfying $B(0)=B(1)=1$ (see $[15,19]$ ). The associated fractional integral is defined by

$$
\left({ }_{a}^{A B} I^{\kappa} \delta\right)(s)=\frac{1-\kappa}{\kappa} \delta(s)+\frac{\kappa}{B(\kappa)}\left({ }_{a} I^{\kappa} \delta\right)(s),
$$

where ${ }_{a} I^{\kappa}$ is the left Riemann-Liouville fractional integral given as

$$
\left({ }_{a} I^{\kappa} \delta\right)(s)=\frac{1}{\Gamma(\kappa)} \int_{a}^{s}(s-v)^{\kappa-1} \delta(v) d \nu .
$$


Consider $d: M \times M \rightarrow[0, \infty)$ given by

$$
d(\delta, \sigma)=\left\|(\delta-\sigma)^{2}\right\|_{\infty}=\sup _{s \in J}(\delta(s)-\sigma(s))^{2},
$$

where $M=C(J, \mathbb{R})$ denotes the set of continuous functions, $(M, d)$ is a complete $b$-metric space with $s_{1}=2$.

We discuss the problem

$$
\begin{aligned}
& \left(\begin{array}{l}
A B C \\
0
\end{array} D^{\kappa} \delta\right)(s)=h(s, \delta(s)), \quad s \in J, 1 \leq \kappa \leq 1, \\
& \delta(0)=\delta_{0},
\end{aligned}
$$

where $D^{\kappa}$ is the Atangana-Baleanu derivative in the Caputo sense of order $\kappa$ and $h: J \times$ $M \rightarrow M$ is continuous with $h(0, \delta(0))=0$.

Proposition 1.5 ([10]) For $0<\kappa<1$, we have

$$
\left({ }^{A B} I_{b}^{K A B C} D^{\kappa} \delta\right)(s)=\delta(s)-\delta(b) .
$$

\section{Main result}

\section{Theorem 2.1 Suppose}

(i) $\exists \omega: \mathbb{R}^{2} \rightarrow \mathbb{R}$ such that

$$
\begin{aligned}
& |h(s, \delta(s))-h(s, \sigma(s))| \\
& \quad \leq \frac{1}{2 \sqrt{2}} \frac{B(\kappa) \Gamma(\kappa)}{(1-\kappa) \Gamma(\kappa)+1} \sqrt{\lambda\left(\gamma\left(|\delta(s)-\sigma(s)|^{2}\right)\right) \gamma\left(|\delta(s)-\sigma(s)|^{2}\right)}
\end{aligned}
$$

for $s \in J, \gamma \in \Omega$, and $\delta, \sigma \in \mathbb{R}$ with $\omega(\delta, \sigma) \geq 0$;

(ii) $\exists \delta_{1} \in C(J)$ with $\omega\left(\delta_{1}(s), T \delta_{1}(s)\right) \geq 0$ for $s \in J$, where $T: C(J) \rightarrow C(J)$ is defined by

$$
(T \delta)(s)=\delta_{0}+{ }_{0}^{A B} I^{K} h(s, \delta(s))
$$

(iii) for $s \in J$ and $\delta, \sigma \in C(J), \omega(\delta(s), \sigma(s)) \geq 0$ implies $\omega(T \delta(s), T \sigma(s)) \geq 0$;

(iv) $\left\{\delta_{n}\right\} \subseteq C(J), \delta_{n} \rightarrow \delta$ in $C(J)$ and $\omega\left(\delta_{n}, \delta_{n+1}\right) \geq 0$, then $\omega\left(\delta_{n}, \delta\right) \geq 0, n \in \mathbb{N}$.

Then problem (6) has at least one solution.

Proof Applying the Atangana-Baleanu integral to both sides of (6) and using Proposition 1.5 , we get

$$
\delta(s)=\delta_{0}+{ }_{0}^{A B} I^{\kappa} h(s, \delta(s)) .
$$

We show that $T$ has a fixed point:

$$
\begin{aligned}
& |T \delta(s)-T \sigma(s)|^{2} \\
& =\left.\left.\right|_{0} ^{A B} I^{\kappa}[h(s, \delta(s))-h(s, \sigma(s))]\right|^{2} \\
& \quad \leq\left|\left[\frac{1-\kappa}{B(\kappa)}[h(s, \delta(s))-h(s, \sigma(s))]+\frac{\kappa}{B(\kappa)} 0 I^{\kappa}[h(s, \delta(s))-h(s, \sigma(s))]\right]\right|^{2}
\end{aligned}
$$




$$
\begin{aligned}
\leq & \left\{\frac{1-\kappa}{B(\kappa)}|h(s, \delta(s))-h(s, \sigma(s))|+\frac{\kappa}{B(\kappa)} I^{\kappa}|h(s, \delta(s))-h(s, \sigma(s))|\right\}^{2} \\
\leq & \left\{\frac{1}{2 \sqrt{2}} \frac{1-\kappa}{B(\kappa)} \times \frac{B(\kappa) \Gamma(\kappa)}{(1-\kappa) \Gamma(\kappa)+1} \sqrt{\lambda\left(\gamma\left(|\delta(s)-\sigma(s)|^{2}\right)\right) \gamma\left(|\delta(s)-\sigma(s)|^{2}\right)}\right. \\
& \left.+\frac{1}{2 \sqrt{2}} \frac{\kappa}{B(\kappa)} \frac{B(\kappa) \Gamma(\kappa)}{(1-\kappa) \Gamma(\kappa)+1} I^{\kappa}(1) \sqrt{\lambda\left(\gamma\left(|\delta(s)-\sigma(s)|^{2}\right)\right) \gamma\left(|\delta(s)-\sigma(s)|^{2}\right)}\right\}^{2} \\
= & \left\{\frac{1}{2 \sqrt{2}} \frac{B(\kappa) \Gamma(\kappa)}{(1-\kappa) \Gamma(\kappa)+1} \sqrt{\lambda\left(\gamma\left(|\delta(s)-\sigma(s)|^{2}\right)\right) \gamma\left(|\delta(s)-\sigma(s)|^{2}\right)}\right\}^{2} \\
& \times\left\{\frac{1-\kappa}{B(\kappa)}+\frac{\kappa}{B(\kappa)} \frac{1}{\kappa \Gamma(\kappa)}\right\}^{2} \\
\leq & \left\{\frac{1}{2 \sqrt{2}} \frac{B(\kappa) \Gamma(\kappa)}{(1-\kappa) \Gamma(\kappa)+1} \sqrt{\lambda\left(\gamma\left(\sup _{s \in J}|\delta(s)-\sigma(s)|^{2}\right)\right) \gamma\left(\sup _{s \in J}|\delta(s)-\sigma(s)|^{2}\right)}\right\}^{2} \\
& \times\left\{\frac{1-\kappa}{B(\kappa)}+\frac{\kappa}{B(\kappa)} \frac{1}{\kappa \Gamma(\kappa)}\right\}^{2} \\
= & \left\{\frac{1}{2 \sqrt{2}} \frac{B(\kappa) \Gamma(\kappa)}{(1-\kappa) \Gamma(\kappa)+1} \sqrt{\lambda(\gamma(d(\delta, \sigma))) \gamma(d(\delta, \sigma))}\right\}^{2} \times\left\{\frac{1-\kappa}{B(\kappa)}+\frac{1}{B(\kappa) \Gamma(\kappa)}\right\}^{2} \\
= & \frac{1}{8} \lambda(\gamma(d(\delta, \sigma))) \gamma(d(\delta, \sigma)) .
\end{aligned}
$$

Hence, for $\delta, \sigma \in C(J), s \in J$ with $\omega(\delta(s), \sigma(s)) \geq 0$, we have

$$
8\left\|(T \delta-T \sigma)^{2}\right\|_{\infty} \leq \lambda(\gamma(d(\delta, \sigma))) \gamma(d(\delta, \sigma)) .
$$

Put $\alpha: C(J) \times C(J) \rightarrow[0, \infty)$ by

$$
\alpha(\delta, \sigma)= \begin{cases}1 & \omega(\delta(s), \sigma(s)) \geq 0 \text { for all } s \in J \\ 0 & \text { else }\end{cases}
$$

and

$$
\begin{aligned}
\alpha(\delta, \sigma) \gamma(8 d(T \delta, T \sigma)) & \leq 8 d(T \delta, T \sigma) \\
& \leq \lambda(\gamma(d(\delta, \sigma))) \gamma(d(\delta, \sigma)) .
\end{aligned}
$$

Then $T$ is an $\alpha-\gamma$-contractive mapping. From (iii),

$$
\begin{aligned}
\alpha(\delta, \sigma) \geq 1 & \Rightarrow \quad \omega(\delta(s), \sigma(s)) \geq 0 \\
& \Rightarrow \quad \omega(T(\delta), T(\sigma)) \geq 0 \\
& \Rightarrow \quad \alpha(T(\delta), T(\sigma)) \geq 1
\end{aligned}
$$

for $\delta, \sigma \in C(J)$. Therefore, $T$ is $\alpha$-admissible. From (ii), there exists $\delta_{0} \in C(J)$ with $\alpha\left(\delta_{0}, T \delta_{0}\right) \geq 1$. By (iv) and Theorem 1.3 , we conclude there exists $\delta^{*} \in C(J)$ with $\delta^{*}=T \delta^{*}$. Hence, $\delta^{*}$ is a solution of the problem.

We denote by $\mathcal{F}$ the family of all functions that satisfy the following conditions: 
(i) $F: \mathbb{R}_{+} \rightarrow \mathbb{R}$ is a strictly increasing mapping;

(ii) $\lim _{n \rightarrow \infty} F\left(\alpha_{n}\right)=-\infty$ if and only if, for each sequence $\left\{\alpha_{n}\right\}_{n \in \mathbb{N}}$ of positive numbers, $\lim _{n \rightarrow \infty} \alpha_{n}=0$;

(iii) there exists $k \in(0,1)$ such that $\lim _{\alpha \rightarrow 0^{+}} \alpha^{k} F(\alpha)=0$.

Definition 2.2 Let $(M, d)$ be a metric space, $g: M \rightarrow M$ is said to be an $\alpha$-type $F$ contraction on $M$ if there exist $\nu>0$ and two functions $F \in \mathcal{F}$ and $\alpha: M \times M \rightarrow\{-\infty\} \cup$ $(0, \infty)$ such that, for all $\delta, \sigma \in M$ satisfying $d(g \delta, g \sigma)>0$, we have

$$
v+\alpha(\delta, \sigma) F(d(g \delta, g \sigma)) \leq F(d(\delta, \sigma))
$$

Theorem 2.3 ([12]) Let $(M, d)$ be a metric space and $g: M \rightarrow M$ be an $\alpha$-type Fcontraction such that:

(i) $\exists \delta_{0} \in M$ with $\alpha\left(\delta_{0}, g \delta_{0}\right) \geq 1$,

(ii) $g$ is $\alpha$-admissible,

(iii) if $\left\{\delta_{n}\right\} \subseteq M$ with $\alpha\left(\delta_{n}, \delta_{n+1}\right) \geq 1$ and $\delta_{n} \rightarrow \delta$, then $\alpha\left(\delta_{n}, \delta\right) \geq 1, n \in N$,

(iv) $F$ is continuous.

Then $g$ has a fixed point $\delta^{*} \in M$ and, for every $\delta_{0} \in M$, the sequence $\left\{g^{n} \delta_{0}\right\}_{n \in N}$ is convergent to $\delta^{*}$.

Theorem 2.4 Suppose

(i) $\exists \omega: \mathbb{R}^{2} \rightarrow \mathbb{R}$ such that

$$
|h(s, \delta(s))-h(s, \sigma(s))| \leq \frac{B(\kappa) \Gamma(\kappa)}{(1-\kappa) \Gamma(\kappa)+1} e^{\frac{-v}{2}}|\delta(s)-\sigma(s)|
$$

for $s \in J$ and $\delta, \sigma \in \mathbb{R}$ with $\omega(\delta, \sigma) \geq 0$;

(ii) $\exists \delta_{1} \in C(J)$ such that $\omega\left(\delta_{1}(s), T \delta_{1}(s)\right) \geq 0$ for $s \in J$, where $T: C(J) \rightarrow C(J)$ is defined by

$$
(T \delta)(s)=\delta_{0}+{ }_{0}^{A B} I^{\kappa} h(s, \delta(s))
$$

(iii) for $s \in J$ and $\delta, \sigma \in C(J), \omega(\delta(s), \sigma(s)) \geq 0$ implies $\omega(T \delta(s), T \sigma(s)) \geq 0$ :

(iv) $\left\{\delta_{n}\right\} \subseteq C(J), \delta_{n} \rightarrow \delta$ in $C(J)$ and $\omega\left(\delta_{n}, \delta_{n+1}\right) \geq 0$, then $\omega\left(\delta_{n}, \delta\right) \geq 0, n \in N$.

Then problem (6) has at least one solution.

Proof Similar to the previous theorem, we demonstrate that $T$ has a fixed point:

$$
\begin{aligned}
\mid T & \delta(s)-\left.T \sigma(s)\right|^{2} \\
& =\left|{ }_{0}^{A B} I^{\kappa}[h(s, \delta(s))-h(s, \sigma(s))]\right|^{2} \\
& \leq\left|\left[\frac{1-\kappa}{B(\kappa)}[h(s, \delta(s))-h(s, \sigma(s))]+\frac{\kappa}{B(\kappa)} 0 I^{\kappa}[h(s, \delta(s))-h(s, \sigma(s))]\right]\right|^{2} \\
& \leq\left\{\frac{1-\kappa}{B(\kappa)}|h(s, \delta(s))-h(s, \sigma(s))|+\frac{\kappa}{B(\kappa)}{ }^{\circ} I^{\kappa}|h(s, \delta(s))-h(s, \sigma(s))|\right\}^{2} \\
& \leq\left\{\frac{1-\kappa}{B(\kappa)} \times \frac{B(\kappa) \Gamma(\kappa)}{(1-\kappa) \Gamma(\kappa)+1} e^{\frac{-v}{2}} \sqrt{|\delta(s)-\sigma(s)|^{2}}\right.
\end{aligned}
$$




$$
\begin{aligned}
& \left.+\frac{\kappa}{B(\kappa)} \frac{B(\kappa) \Gamma(\kappa)}{(1-\kappa) \Gamma(\kappa)+1} I^{\kappa}(1) e^{\frac{-v}{2}} \sqrt{|\delta(s)-\sigma(s)|^{2}}\right\}^{2} \\
= & \left\{\frac{B(\kappa) \Gamma(\kappa)}{(1-\kappa) \Gamma(\kappa)+1} e^{\frac{-v}{2}} \sqrt{|\delta(s)-\sigma(s)|^{2}}\right\}^{2} \\
& \times\left\{\frac{1-\kappa}{B(\kappa)}+\frac{\kappa}{B(\kappa)} \frac{1}{\kappa \Gamma(\kappa)}\right\}^{2} \\
\leq & \left\{\frac{B(\kappa) \Gamma(\kappa)}{(1-\kappa) \Gamma(\kappa)+1} e^{\frac{-v}{2}} \sqrt{\sup _{s \in J}|\delta(s)-\sigma(s)|^{2}}\right\}^{2} \\
& \times\left\{\frac{1-\kappa}{B(\kappa)}+\frac{\kappa}{B(\kappa)} \frac{1}{\kappa \Gamma(\kappa)}\right\}^{2} \\
= & \left\{\frac{B(\kappa) \Gamma(\kappa)}{(1-\kappa) \Gamma(\kappa)+1} e^{\frac{-v}{2}} \sqrt{d(\delta, \sigma)}\right\}^{2} \times\left\{\frac{1-\kappa}{B(\kappa)}+\frac{1}{B(\kappa) \Gamma(\kappa)}\right\}^{2} \\
= & e^{-v} d(\delta, \sigma) .
\end{aligned}
$$

Hence, for $\delta, \sigma \in C(J), s \in J$ with $\omega(\delta(s), \sigma(s)) \geq 0$, we have

$$
d(T \delta, T \sigma) \leq e^{-v} d(\delta, \sigma) .
$$

So

$$
\ln (d(T \delta, T \sigma)) \leq \ln \left(e^{-v} d(\delta, \sigma)\right)
$$

therefore

$$
v+\ln (d(T \delta, T \sigma)) \leq \ln (d(\delta, \sigma)) .
$$

Now, let $F:[0, \infty) \rightarrow R$ given by $F(u)=\ln u, u>0$, then $F \in \mathcal{F}$.

Put $\alpha: C(J) \times C(J) \rightarrow\{-\infty\} \cup[0, \infty)$ by

$$
\alpha(\delta, \sigma)= \begin{cases}1 & \omega(\delta(s), \sigma(s)) \geq 0 \text { for all } s \in J \\ -\infty & \text { else }\end{cases}
$$

Therefore $v+\alpha(\delta, \sigma) F(d(T \delta, T \sigma)) \leq F(d(\delta, \sigma))$ for $\delta, \sigma \in M$ with $d(T \delta, T \sigma)>0$. For this reason, $T$ is an $\alpha$-type $F$-contraction. From (iii),

$$
\begin{aligned}
\alpha(\delta, \sigma) \geq 1 & \Rightarrow \quad \omega(\delta(s), \sigma(s)) \geq 0 \\
& \Rightarrow \quad \omega(T(\delta), T(\sigma)) \geq 0 \\
& \Rightarrow \quad \alpha(T(\delta), T(\sigma)) \geq 1
\end{aligned}
$$

for all $\delta, \sigma \in C(J)$. Thus, $T$ is $\alpha$-admissible. From (ii), there exists $\delta_{0} \in C(J)$ with $\alpha\left(\delta_{0}, T \delta_{0}\right) \geq 1$. By (iv) and Theorem 2.3, we conclude $\delta^{*} \in C(J)$ with $\delta^{*}=T \delta^{*}$. Hence, $\delta^{*}$ is a solution of the problem.

Now let $\mathcal{F}$ be the set of functions $g:(0, \infty) \rightarrow \mathbb{R}$ with the conditions: 
$\left(\mathcal{F}_{1}\right)$ If $0<s<t$, then $g(s) \leq g(t)$;

$\left(\mathcal{F}_{2}\right)$ If $\left\{s_{n}\right\} \subset(0,+\infty)$, then

$$
\lim _{n \rightarrow+\infty} s_{n}=0 \quad \text { if and only if } \lim _{n \rightarrow+\infty} g\left(s_{n}\right)=-\infty .
$$

The space of an $\mathcal{F}$-metric is defined as follows.

Definition 2.5 ([36]) Let $M$ be nonempty, $d: M \times M \rightarrow[0,+\infty)$ and $(g, a) \in \mathcal{F} \times[0,+\infty)$ such that

$\left(d_{1}\right)(\delta, \sigma) \in M \times M, d(\delta, \sigma)=0 \Leftrightarrow \delta=\sigma ;$

$\left(d_{2}\right) d(\delta, \sigma)=d(\sigma, \delta)$, for $(\delta, \sigma) \in M \times M$;

(d) For $(\delta, \sigma) \in M \times M, N \in \mathbb{N}, N \geq 2$, and for $\left(u_{i}\right)_{i=1}^{N} \subset M$ with $\left(u_{1}, u_{N}\right)=(\delta$, $\sigma)$, we have

$$
d(\delta, \sigma)>0 \quad \text { implies } \quad g(d(\delta, \sigma)) \leq g\left(\sum_{i=1}^{N-1} d\left(u_{i}, u_{i+1}\right)\right)+a \text {. }
$$

Then $d$ is said to be an $\mathcal{F}$-metric on $M$, and the pair $(M, d)$ is said to be an $\mathcal{F}$-metric space.

A sequence $\left\{\delta_{n}\right\}$ in $(M, d)$ is convergent to $\delta$ with respect to the $\mathcal{F}$-metric $d$ if

$$
\lim _{n \rightarrow \infty} d\left(\delta_{n}, \delta\right)=0
$$

A sequence $\left\{\delta_{n}\right\}$ in $(M, d)$ is called $\mathcal{F}$-Cauchy if

$$
\lim _{n, m \rightarrow+\infty} d\left(\delta_{n}, \delta_{m}\right)=0
$$

$(M, d)$ is $\mathcal{F}$-complete if every $\mathcal{F}$-Cauchy sequence in $M$ is $\mathcal{F}$-convergent to a specified element in $M$. Let $\Gamma$ be the set of functions $\gamma:[0, \infty) \rightarrow[0, \infty)$ such that

$\left(\gamma_{1}\right) \gamma$ is nondecreasing;

$\left(\gamma_{2}\right) \sum_{n=1}^{\infty} \gamma^{n}(s)<\infty$ for $s \in \mathbb{R}^{+}$, where $\gamma^{n}$ is the $n$th iterate of $\gamma$.

Definition 2.6 ([37]) Let $\alpha: M \times M \rightarrow[0, \infty)$, then $g: M \rightarrow M$ is said to be an $\alpha$-orbital admissible if, for $s \in M$, we have

$$
\alpha(s, g s) \geq 1 \quad \Rightarrow \quad \alpha\left(g s, g^{2} s\right) \geq 1
$$

Theorem $2.7([9])$ Assume $(M, d)$ to be an $\mathcal{F}$-complete metric space and $g: M \rightarrow M$ such that

$$
\alpha(\delta, \sigma) d(g \delta, g \sigma) \leq \gamma(d(\delta, \sigma))
$$

for $\delta, \sigma \in M$, where $\gamma \in \Gamma$. Suppose

(i) $g$ is orbital $\alpha$-admissible;

(ii) there exists $\delta_{0} \in M$ with $\alpha\left(\delta_{0}, g \delta_{0}\right) \geq 1$;

(iii) $g \in \mathcal{F}$ verifying $\left(d_{3}\right)$ is assumed to be continuous; also, $\gamma$ is chosen to be continuous and to satisfy that $g(u)>g(\gamma(u))+a, u \in(0, \infty)$, where a is also given in $\left(d_{3}\right)$;

then $f$ has a fixed point. 
Consider the $\mathcal{F}$-metric $d: M \times M \rightarrow[0, \infty)$ with $M=C(J, \mathbb{N})$, given as

$$
d(\delta, \sigma)= \begin{cases}e^{|\delta-\sigma|} & \text { if } \delta \neq \sigma, \\ 0 & \text { if } \delta=\sigma\end{cases}
$$

where $M=\{0,1,2, \ldots\}, g(s)=-\frac{1}{s}$ for $s>0, a=1$ and $g$ is continuous on $(0, \infty)$. The condition $g(u)>g(\gamma(u))+a, u>0$, becomes $-\frac{1}{u}>\frac{1}{\gamma(u)}>1$, that is, $\gamma$ is chosen to be continuous such that

$$
\gamma(u)<\frac{u}{u+1} .
$$

Also consider that $\gamma$ satisfies the following additional condition:

$$
e^{\gamma(s)} \leq \gamma\left(e^{s}\right), \quad s \in\{0,1,2,3, \ldots\}
$$

\section{Theorem 2.8 Assume}

(i) $\exists \omega: \mathbb{R}^{2} \rightarrow \mathbb{R}$ with

$$
|h(s, \delta(s))-h(s, \sigma(s))| \leq \frac{B(\kappa) \Gamma(\kappa)}{(1-\kappa) \Gamma(\kappa)+1} \gamma(|\delta(s)-\sigma(s)|)
$$

for $s \in J$ and $\delta, \sigma \in \mathbb{R}$ with $\omega(\delta, \sigma) \geq 0$;

(ii) $\exists \delta_{1} \in C(J)$ with $\omega\left(\delta_{1}(s), T \delta_{1}(s)\right) \geq 0$ for $s \in J$, where $T: C(J) \rightarrow C(J)$ is defined by

$$
(T \delta)(s)=\delta_{0}+{ }_{0}^{A B} I^{K} h(s, \delta(s))
$$

(iii) for $s \in J$ and $\delta \in C(J), \omega(\delta(s), T \delta(s)) \geq 0$ implies $\omega\left(T \delta(s), T^{2} \delta(s)\right) \geq 0$.

Then (6) has at least one solution.

Similar to the previous theorem, we demonstrate that $T$ has a fixed point:

$$
\begin{aligned}
&|T \delta(s)-T \sigma(s)| \\
&=\left|{ }_{0}^{A B} I^{\kappa}[h(s, \delta(s))-h(s, \sigma(s))]\right| \\
& \leq \mid\left[\frac{1-\kappa}{B(\kappa)}[h(s, \delta(s))-h(s, \sigma(s))]+\frac{\kappa}{B(\kappa)}{ }^{\kappa} I^{\kappa}[h(s, \delta(s))-h(s, \sigma(s))]\right] \\
& \leq\left\{\frac{1-\kappa}{B(\kappa)}|h(s, \delta(s))-h(s, \sigma(s))|+\frac{\kappa}{B(\kappa)}{ }_{0} I^{\kappa}|h(s, \delta(s))-h(s, \sigma(s))|\right\} \\
& \leq\left\{\frac{1-\kappa}{B(\kappa)} \times \frac{B(\kappa) \Gamma(\kappa)}{(1-\kappa) \Gamma(\kappa)+1} \gamma(|\delta(s)-\sigma(s)|)\right\} \\
&+\left\{\frac{\kappa}{B(\kappa)} \frac{B(\kappa) \Gamma(\kappa)}{(1-\kappa) \Gamma(\kappa)+1}{ }_{0} I^{\kappa}(1) \gamma(|\delta(s)-\sigma(s)|)\right\} \\
&=\left\{\frac{B(\kappa) \Gamma(\kappa)}{(1-\kappa) \Gamma(\kappa)+1} \gamma(|\delta(s)-\sigma(s)|)\right\}\left\{\frac{1-\kappa}{B(\kappa)}+\frac{\kappa}{B(\kappa)} \frac{1}{\kappa \Gamma(\kappa)}\right\} \\
&= \gamma(|\delta(s)-\sigma(s)|) .
\end{aligned}
$$


Hence, for $\delta, \sigma \in C(J), s \in J$ with $\omega(\delta(s), \sigma(s)) \geq 0$, we have

$$
d(T \delta, T \sigma)=e^{|T \delta(s)-T \sigma(s)|} \leq e^{\gamma(|\delta(s)-\sigma(s)|)} \leq \gamma\left(e^{|\delta(s)-\sigma(s)|}\right)=\gamma(d(\delta, \sigma))
$$

Put $\alpha: C(J) \times C(J) \rightarrow[0, \infty)$ by

$$
\alpha(\delta, \sigma)= \begin{cases}1 & \omega(\delta(s), \sigma(s)) \geq 0 \text { for all } s \in J \\ 0 & \text { else. }\end{cases}
$$

Therefore $\alpha(\delta, \sigma) d(T \delta, T \sigma) \leq d(T \delta, T \sigma) \leq \gamma(d(\delta, \sigma))$ for all $\delta, \sigma \in M$ with $d(T \delta, T \sigma)>0$. From (iii),

$$
\begin{aligned}
\alpha(\delta, T \delta) \geq 1 & \Rightarrow \quad \omega(\delta(s), T \delta(s)) \geq 0 \\
& \Rightarrow \quad \omega\left(T(\delta), T^{2}(\delta)\right) \geq 0 \\
& \Rightarrow \quad \alpha\left(T(\delta), T^{2}(\delta)\right) \geq 1
\end{aligned}
$$

for $\delta \in C(J)$. Thus, $T$ is orbital $\alpha$-admissible. From (ii), there exists $\delta_{1} \in C(J)$ with $\alpha\left(\delta_{1}, T \delta_{1}\right) \geq 1$. By (iii) and Theorem 2.7, we get $\sigma^{*} \in C(J)$ with $\delta^{*}=T \delta^{*}$. Hence, $\delta^{*}$ is a solution of the problem.

\section{Conclusion}

In this manuscript, we extend some of the fractional differential equations of RiemannLiouville and Caputo type to the fractional differential equations of Atangana-Baleanu in the Caputo sense.

\section{Acknowledgements}

Not applicable.

Funding

Not applicable.

Availability of data and materials

Data sharing not applicable to this article as no datasets were generated or analyzed during the current study.

\section{Competing interests}

The authors declare that they have no competing interests.

\section{Authors' contributions}

All authors read and approved the final manuscript.

\section{Author details}

${ }^{1}$ Department of Mathematics, Faculty of Basic Science, University of Bonab, Bonab, Iran. ${ }^{2}$ Department of Mathematics, Cankaya University, Ankara, Turkey. ${ }^{3}$ Institute of Space Sciences, Magurele-Bucharest, Romania.

\section{Publisher's Note}

Springer Nature remains neutral with regard to jurisdictional claims in published maps and institutional affiliations.

Received: 15 November 2019 Accepted: 17 March 2020 Published online: 30 March 2020

\section{References}

1. Afshari, H., Aydi, H., Karapınar, E.: On generalized $\alpha$ - $\psi$-Geraghty contractions on b-metric spaces. Georgian Math. J. 27(1), 9-21 (2020). https://doi.org/10.1515/gmj-2017-0063

2. Afshari, H., Aydi, H., Karapınar, E.: Existence of fixed points of set-valued mappings in b-metric spaces. East Asian Math. J. 32(3), 319-332 (2016) 
3. Afshari, $\mathrm{H}$.: Solution of fractional differential equations in quasi- $b$-metric and $b$-metric-like spaces. Adv. Differ. Equ. $2018,285(2018)$

4. Afshari, H., Kalantari, S., Karapınar, E.: Solution of fractional differential equations via coupled fixed point. Electron. J. Differ. Equ. 2015, 286 (2015)

5. Afshari, H., Marasi, H.R., Aydi, H.: Existence and uniqueness of positive solutions for boundary value problems of fractional differential equations. Filomat 31, 2675-2682 (2017). https://doi.org/10.2298/FIL1709675A

6. Ajou, A., Oqielat, M.N., Zhour, Z.A., Kumar, S., Momani, S.: Solitary solutions for time-fractional nonlinear dispersive PDEs in the sense of conformable fractional derivative. Chaos 29, 093102 (2019)

7. Alqahtani, B., Fulga, A., Jarad, F., Karapınar, E.: Nonlinear F-contractions on b-metric spaces and differential equations in the frame of fractional derivatives with Mittag-Leffler kernel. Chaos Solitons Fractals 128, 349-354 (2019). https://doi.org/10.1016/j.chaos.2019.08.002

8. Atangana, A., Baleanu, D.: New fractional derivative with non-local and non-singular kernel. Therm. Sci. 20, 757-763 (2016)

9. Aydi, H., Karapınar, E., Mitrovi, Z.D., Rashid, T.: A remark on "Existence and uniqueness for a neutral differential problem with unbounded delay via fixed point results F-metric space". Rev. R. Acad. Cienc. Exactas Fís. Nat., Ser. A Mat. (2019). https://doi.org/10.1007/s13398-019-00690-9

10. Abdeljawad, T., Baleanu, D.: Integration by parts and its applications of a new nonlocal fractional derivative with Mittag-Leffler nonsingular kernel. J. Nonlinear Sci. Appl. 10, 1098-1107 (2017)

11. Doungmo Goufoa, E.F., Kumar, S., Mugisha, S.B.: Similarities in a fifth-order evolution equation with and with no singular kernel. Chaos Solitons Fractals 130, 109467 (2020)

12. Gopal, D., Abbas, M., Kumar, D.P., Vetro, C.: Fixed points of $\alpha$-type F-contractive mappings with an application to nonlinear fractional differential equation. Acta Math. Sci. 36(3), 957-970 (2016)

13. Odibat, Z., Kumar, S.: A robust computational algorithm of homotopy asymptotic method for solving systems of fractional differential equation. J. Comput. Nonlinear Dyn. 14(8), 081004 (2019)

14. Osler, T.J.: Fractional derivatives and Leibniz rule. Am. Math. Mon. 78, 645-649 (1971)

15. Kilbas, A., Srivastava, H.M., Trujillo, J.J.: Theory and Application of Fractional Differential Equations. North Holland Math. Stud., vol. 204 (2006)

16. Kumar, S., Momani, S., Aldhaifalla, M., Nisar, K.S.: Numerical solutions of nonlinear fractional model arising in the appearance of the strip patterns in two-dimensional systems. Adv. Differ. Equ. 2019, 413 (2019)

17. Samei, M.E., Hedayati, V., Ranjbar, G.K.: The existence of solution for k-dimensional system of Langevin Hadamard-type fractional differential inclusions with $2 k$ different fractional orders. Mediterr. J. Math. 17, 37 (2020) https://doi.org/10.1007/s00009-019-1471-2

18. Samei, M.E., Hedayati, V., Rezapour, S.: Existence results for a fraction hybrid differential inclusion with Caputo-Hadamard type fractional derivative. Adv. Differ. Equ. 2019, 163 (2019). https://doi.org/10.1186/s13662-019-2090-8

19. Samko, S.G., Kilbas, A.A., Marichev, O.: Fractional Integrals and Derivatives: Theory and Applications. Gordon \& Breach, Yverdon (1993)

20. Owolabi, K.M.: Behavioural study of symbiosis dynamics via the Caputo and Atangana-Baleanu fractional derivatives. Chaos Solitons Fractals 122, 89-101 (2019). https://doi.org/10.1016/j.chaos.2019.03.014

21. Owolabi, K.M.: Modelling and simulation of a dynamical system with the Atangana-Baleanu fractional derivative. Eur. Phys. J. Plus 133, 15 (2018)

22. Owolabi, K.M., Atangana, A.: Computational study of multi-species fractional reaction-diffusion system with $A B C$ operator. Chaos Solitons Fractals 128, 280-289 (2019). https://doi.org/10.1016/j.chaos.2019.07.050

23. Owolabi, K.M., Atangana, A.: Mathematical analysis and computational experiments for an epidemic system with nonlocal and nonsingular derivative. Chaos Solitons Fractals 126, 41-49 (2019). https://doi.org/10.1016/j.chaos.2019.06.001

24. Owolabi, K.M., Atangana, A.: On the formulation of Adams-Bashforth scheme with Atangana-Baleanu-Caputo fractional derivative to model chaotic problems. Chaos 29, 023111 (2019). https://doi.org/10.1063/1.5085490

25. Owolabi, K.M., Gomez-Aguilar, J.F., Karaagac, B.: Modelling, analysis and simulations of some chaotic systems using derivative with Mittag-Leffler kernel. Chaos Solitons Fractals 125, 54-63 (2019). https://doi.org/10.1016/j.chaos.2019.05.019

26. Owolabi, K.M., Hammouch, Z: Mathematical modeling and analysis of two-variable system with noninteger-order derivative. Chaos 29, 013145 (2019). https://doi.org/10.1063/1.5086909

27. Owolabi, K.M., Pindza, E.: Modeling and simulation of nonlinear dynamical system in the frame of nonlocal and non-singular derivatives. Chaos Solitons Fractals 125, 54-63 (2019). https://doi.org/10.1016/j.chaos.2019.05.019

28. Jarad, F., Abdeljawad, T., Hammouch, Z:: On a class of ordinary differential equations in the frame of Atangana-Baleanu fractional derivative. Chaos Solitons Fractals 117, 16-20 (2018)

29. Ravichandran, C., Logeswari, K., Jarad, F.: New results on existence in the framework of Atangana-Baleanu derivative for fractional integro-differential equations. Chaos Solitons Fractals 125, 194-200 (2019)

30. Avalos-Ruiza, L.F., Gomez-Aguilar, J.F., Atangana, A., Owolabi, K.M.: On the dynamics of fractional maps with power-law, exponential decay and Mittag-Leffler memory. Chaos Solitons Fractals 127, 364-388 (2019). https://doi.org/10.1016/j.chaos.2019.07.010

31. Karapınar, E., Abdeljawad, T., Jarad, F.: Applying new fixed point theorems on fractional and ordinary differential equations. Adv. Differ. Equ. 2019, 421 (2019)

32. Shoaib, M., Abdeljawad, T., Sarwar, M., Jarad, F.: Fixed point theorems for multi-valued contractions in $b$-metric spaces with applications to fractional differential and integral equations. IEEE Access 7, 127373-127383 (2019)

33. Samet, B., Vetro, C., Vetro, P.: Fixed point theorems for $\alpha$ - $\psi$-contractive type mappings. Nonlinear Anal. 75 , 2154-2165 (2012)

34. Karapınar, E., Samet, B.: Generalized $\alpha$ - $\psi$-contractive type mappings and related fixed point theorems with applications. Abstr. Appl. Anal. 2012, Article ID 793486 (2012)

35. Wardowski, D.: Fixed point theory of a new type of contractive mappings in complete metric spaces. Fixed Point Theory Appl. 2012, 94 (2012) 
36. Jleli, M., Samet, B.: On a new generalization of metric spaces. Fixed Point Theory Appl. 2018, 128 (2018)

37. Popescu, O.: Some new fixed point theorems for $\alpha$-Geraghty-contraction type maps in metric spaces. Fixed Point Theory Appl. 2014, 190 (2014)

Submit your manuscript to a SpringerOpen ${ }^{\odot}$ journal and benefit from:

- Convenient online submission

- Rigorous peer review

- Open access: articles freely available online

- High visibility within the field

- Retaining the copyright to your article

Submit your next manuscript at $\gg$ springeropen.com 